\title{
Random matrix theory in semiclassical quantum mechanics of chaotic systems
}

\author{
Michael Wilkinson \\ Department of Physics and Applied Physics, John Anderson Building, University of \\ Strathclyde, Glasgow G4 0NG, UK
}

Received 1 June 1987

\begin{abstract}
The statistical properties of the spectrum of systems which have a chaotic classical limit have been found to be similar to those of random matrix ensembles. This paper will explain this correspondence, including the fact that long-ranged spectral statistics show deviations from the results of random matrix theory.

The method depends on the ambiguity of quantisation of a given classical system: although the energy levels depend on the particular quantisation used, the spectral statistics are assumed to depend only on the classical motion. The ambiguity of quantisation can be represented by a small perturbation acting on the Hamiltonian. This perturbation executes a random walk, which causes its matrix elements to undergo a random walk. If the matrix elements are completely uncorrelated, the spectral statistics are those of random matrix theory. In the case of classically chaotic systems, the matrix elements are constrained by sum rules related to classical periodic orbits. This leads to deviations of the long-ranged spectral statistics from the predictions of random matrix theory.
\end{abstract}

\section{Introduction}

There is now a great deal of evidence that the statistical properties of the semiclassical spectrum of systems with a chaotic classical limit ( $K$ systems) are similar to those of random matrix ensembles. The evidence for this correspondence is discussed by Berry (1983) and Bohigas et al (1984). When there are classical constants of motion, the spectral statistics are very different. The case of completely integrable motion in particular is thoroughly understood (Berry and Tabor 1976, 1977a, b). The correspondence between the spectral statistics of classically chaotic systems and those of random matrices is not complete. It is known that the short-ranged spectral statistics, those depending only on energy levels separated by a few multiples of the mean level spacing, show excellent agreement with random matrix theory. Long-ranged spectral statistics, depending on energy levels separated by $o(\hbar / \tau)$, where $\tau$ is some time characterising the classical motion, show deviations from the predictions of random matrix theory (Berry 1985).

The deviations from the results of random matrix theory exhibited by long-ranged statistics are, in principle, fully understood. These statistics depend on the smoothed density of states, for which a semiclassical theory, due to Gutzwiller $(1967,1969,1970$, $1971,1980)$ and Balian and Bloch $(1972,1974)$ already exists, relating fluctuations in 
the smoothed density of states to periodic orbits of the classical motion. The correspondence between the short-ranged spectral statistics and the results of random matrix theory has not been fully explained. In an important paper, Hannay and Ozorio de Almeida (1984) established a useful sum rule for the periodic orbit corrections to the smoothed density of states, and used this result to calculate the tail of the two-level correlation function. This work has been extended by Berry (1985), who used their result to calculate the $\Delta_{3}$ statistic (Dyson and Mehta 1963). Pechukas (1983) proposed a theory which attempts a general explanation of the correspondence with random matrix theory, but this theory contains assumptions which are hard to justify and does not explain the deviations exhibited by the long-ranged statistics.

This paper presents a new theory which explains both the correspondence of the short-ranged statistics with random matrix theory, and the deviations shown by longranged statistics. It is based on two key ideas. Firstly, the theory relies on the fact that quantisation (assigning a Hermitian operator to a given classical system) is ambiguous. This ambiguity of quantisation can be represented by a small perturbation $\hat{A}$ added to the Hamiltonian $\hat{H}_{0}$. This perturbation is of size $o\left(\hbar^{2}\right)$. This should be compared with the mean level spacing, which is $o\left(\hbar^{d}\right)$ for a system of $d$ degrees of freedom. When $d \geqslant 2$ (which is the case for all chaotic systems with a time-independent Hamiltonian) this perturbation can be large enough to shift energy levels by many times their mean separation. The energy levels themselves are not determined by the classical motion, but for almost all choices of quantisation the spectral statistics depend only on the classical motion. Often (e.g. when the Hamiltonian is of the form $H=\frac{1}{2} p^{2}+$ $V)$ there is a particular quantisation which is physically correct. It is assumed that the statistics of this preferred quantisation are typical. Numerical evidence suggests that this assumption is usually correct, but the hyperbolic map of a torus appears to be one example where the physically preferred quantisation (Hannay and Berry 1980) is not typical.

The second key idea is to make the perturbation $\hat{A}$ execute a random walk. This causes its matrix elements $A_{n m}$ to undergo a random walk. This method was used by Dyson (1962b) to rederive many of the results of random matrix theory. In the case treated by Dyson the matrix elements are completely uncorrelated. In the problem considered here, the matrix elements are locally uncorrelated, but collectively they are constrained by a semiclassical sum rule related to classical periodic orbits. This explains why the long-ranged statistics differ from the results of random matrix theory.

The ambiguity of quantisation is discussed in greater detail in appendix 1 , where it is shown that this ambiguity can be represented by a small perturbation of the Hamiltonian. Section 2 discusses the effect of making this perturbation execute a random walk in function space. If there are no constants of motion, the matrix elements of this perturbation (in a basis of eigenstates of the system) undergo uncorrelated random walks, and the resulting motion of the energy levels is governed by Dyson's Brownian motion model. Section 3 describes three important sum rules, which relate matrix elements of the perturbation to properties of the classical motion. It is shown that, although the diagonal matrix elements are locally uncorrelated, they are collectively constrained by a sum rule related to classical periodic orbits. Section 4 discusses the implications of this result, which explains the deviations from the spectral statistics of the usual random matrix models. The theory does not apply if the perturbation does not destroy any constants of motion of the Hamiltonian; $\S 5$ explains why this excludes classically integrable systems. Finally $\S 6$ is a summary and conclusion. 


\section{A Brownian motion model for spectral statistics}

In appendix 1 it is demonstrated that the ambiguity involved in quantising a classical Hamiltonian $H(\boldsymbol{q}, \boldsymbol{p})$ can be represented by a small perturbation $A(\boldsymbol{q}, \boldsymbol{p})$ added to the Hamiltonian. If the system has time reversal symmetry, the perturbation should respect this symmetry, but apart from this constraint $A(q, p)$ can be regarded as an arbitrary perturbation. The perturbation is large enough to shift the energy levels significantly, but the statistical properties of the spectrum should be the same for almost all quantisations. A convenient way to calculate these statistical properties is to make the perturbation undergo a random walk. This idea was used by Dyson (1962b) to derive a statistical model for the spectrum in which mutually repelling particles (energy levels) embedded in a viscous medium undergo Brownian motion. This section will discuss Dyson's Brownian motion model in the context of semiclassical quantum mechanics.

The perturbation $A(q, p)$ depends on a fictitious time variable $\Gamma$, and $A(\Gamma)$ executes a random walk. This random walk would be realised by expanding $A(q, p ; \Gamma)$ in a very large basis set

$$
A(\boldsymbol{q}, \boldsymbol{p} ; \Gamma)=\sum_{i} C_{i}(\Gamma) a_{i}(\boldsymbol{q}, \boldsymbol{p})
$$

and making the coefficients $C_{i}(\Gamma)$ undergo independent random walks, so that

$$
\left\langle C_{i}\right\rangle=0 \quad\left\langle C_{i} C_{j}\right\rangle=\Gamma \delta_{i j}
$$

where $\langle x\rangle$ denotes the ensemble average of $x$. The basis set should be chosen so that any function which does not vary faster than some very small length scale $\tau_{0}$ can be reproduced by the expansion (2.1), but all of the basis functions $a_{i}$ should be analytic. Note that a typical function drawn from the ensemble will be a rapidly oscillating function with fine detail on length scales down to $\tau_{0}$.

There is a quantum perturbation $\hat{A}$ corresponding to the classical perturbation $\boldsymbol{A}(\boldsymbol{q}, \boldsymbol{p})$. Consider the matrix elements $\boldsymbol{A}_{n m}=\langle\boldsymbol{n}|\hat{\boldsymbol{A}}| m\rangle$, where $|n\rangle,|\boldsymbol{m}\rangle$ are eigenstates of some initial quantisation $\hat{H}_{0}$ of $H(\boldsymbol{q}, \boldsymbol{p})$. We assume that $\hat{A}$ does not respect any symmetries of $\hat{H}_{0}$. In the case of a chaotic system this assumption is justified, but if $H(q, p)$ is integrable the assumption is false; the reasons for this are discussed in $\S 5$. Provided $\hat{A}$ does not respect any symmetry of $\hat{H}_{0}$, the matrix elements $A_{n m}$ are those of a random operator, $\hat{A}$, in an arbitrary basis. Random matrix theory (Porter 1965) shows that the matrix elements are Gaussian distributed and uncorrelated. The mean and variance depend on whether the matrix element is diagonal or off-diagonal:

$$
\begin{aligned}
& \left\langle A_{n m}\right\rangle^{\prime}=0 \quad\left\langle\left|A_{n m}\right|^{2}\right\rangle^{\prime}=\sigma^{2} \\
& \left\langle A_{n n}\right\rangle^{\prime}=f_{0} \quad\left\langle\left(A_{n n}-f_{0}\right)^{2}\right\rangle^{\prime}=\beta \sigma^{2}
\end{aligned}
$$

where $(X)^{\prime}$ denotes the average of $X$ over the matrix. The ensemble average of $f_{0}$ is zero:

$$
\left\langle f_{0}\right\rangle=0
$$

and the constant $\beta$ depends on whether $\hat{A}$ has time reversal symmetry (GOE case) or not (GUE case) (see Porter (1965) for discussion of Gaussian orthogonal and Gaussian unitary ensembles):

$$
\beta= \begin{cases}1 & \text { GUE } \\ 2 & \text { GOE }\end{cases}
$$


Note that these conclusions do not depend on any assumptions about the nature of the eigenstates $|n\rangle$ and $|m\rangle$, which are eigenstates of $\hat{H}_{0}$, and have nothing to do with the perturbation $\hat{A}$. Because of $(2.2)$, the variance $\sigma^{2}$ increases linearly with the fictitious time $\Gamma$

$$
\sigma^{2}=\kappa \Gamma .
$$

There are minor corrections to this picture which arise because the perturbation $\hat{A}$ has an analytic classical limit $\boldsymbol{A}(\boldsymbol{q}, \boldsymbol{p})$. Firstly, the (locally averaged) variance $\sigma^{2}$ is not constant, but depends on the energies $E_{n}, E_{m}$ of the states $|n\rangle,|m\rangle$; similarly $f_{0}$ depends on $E_{m}$. Secondly, although the diagonal matrix elements are locally uncorrelated, they are collectively constrained by a sum rule. Both of these corrections will be discussed in detail in $\$ 3$.

Now consider the effect of the perturbation $\hat{A}$ on an energy level $E_{n}$. The shift in the energy level due to the perturbation is

$$
\delta E_{n}=A_{n n}+\sum_{m \neq n} \frac{\left|A_{n m}\right|^{2}}{E_{n}-E_{m}}+o\left(A^{3}\right) .
$$

The ensemble average and variance of this energy shift are, respectively

$$
\left\langle\delta E_{n}\right\rangle=\left\langle f_{0}\right\rangle+\sigma^{2} \sum_{m \neq n} \frac{1}{E_{n}-E_{m}}=\kappa \delta \Gamma \sum_{m \neq n} \frac{1}{E_{n}-E_{m}}
$$

and

$$
\left\langle\left(\delta E_{n}-f_{0}\right)^{2}\right\rangle=\left\langle\left(A_{n n}-f_{0}\right)^{2}\right\rangle=\beta \sigma^{2}=\beta \kappa \delta \Gamma
$$

to lowest order in $\delta \Gamma$. Note that, because $\hat{A}$ executes a random walk, both the mean and variance contain contributions of size $o(\delta \Gamma)$. Equations $(2.8),(2.9)$ have an interesting physical interpretation. Equation (2.8) describes particles with positions $E_{n}$ moving in a viscous medium, where the force $F_{n}$ is equal to the viscosity $\nu$ times the velocity

$$
\nu\left\langle\delta E_{n}\right\rangle=F_{n} \delta \Gamma
$$

and equation (2.9) can be interpreted as a Brownian motion of the particles, with diffusion constant $D$ :

$$
\left\langle\left(\delta E_{n}-f_{0}\right)^{2}\right\rangle=D \delta \Gamma .
$$

The mutually repulsive force $F_{n}$ and diffusion constant $D$ are given by

$$
\frac{F_{n}}{\nu}=\kappa \sum_{m \neq n} \frac{1}{E_{n}-E_{m}} \quad D=\beta \kappa .
$$

Dyson (1962b) showed that the equilibrium distribution of the energies $E_{n}$ resulting from this motion has the same statistics as the energy level distribution of random matrix theory.

\section{Sum rules for matrix elements}

In $\S 2$ the effect of the perturbation $\hat{A}$ representing the ambiguity of quantisation was discussed. Random matrix theory shows that the matrix elements of $\hat{A}$ are locally uncorrelated and Gaussian distributed. The only constraints on $\hat{A}$ are that it has an 
analytic (but usually rapidly varying) classical limit $A(\boldsymbol{q}, \boldsymbol{p})$, and that if $H(\boldsymbol{q}, \boldsymbol{p})$ has time reversal symmetry, $\hat{A}$ respects this symmetry. In this section it will be shown how these constraints lead to three important sum rules for the matrix elements of $\hat{A}$.

The first sum rule involves the off-diagonal matrix elements $A_{n m}$, and gives the variance of their distribution, $\sigma^{2}$, in terms of classical quantities.

The second sum rule concerns the diagonal matrix elements $A_{n n}$, and gives the average value $f$ of $A_{n n}$ over a short energy interval of length $\varepsilon$, for a fixed perturbation $\hat{A}$ (this should not be confused with the ensemble average, which is zero). This average is expressed as a sum of which each term corresponds to a classical periodic orbit. This sum rule shows that the diagonal matrix elements $A_{n n}$ are not completely uncorrelated; large numbers of matrix elements are collectively constrained by this sum rule. The important consequences of this are discussed in $\$ 4$.

A third sum rule is calculated as a check on the consistency of the results. If the diagonal matrix elements are locally uncorrelated, as predicted by random matrix theory, then the variance of the average $f$ should satisfy $\left\langle f^{2}\right\rangle \propto \varepsilon^{-1}$ in the limit $\varepsilon \rightarrow 0$. The sum rule of Hannay and Ozorio de Almeida (1984) is adapted to show that the periodic orbit sum for $f$ does satisfy this condition.

\subsection{Off-diagonal matrix elements}

Consider the sum

$$
S(E, \Delta E)=\sum_{n m}\left|A_{n m}\right|^{2} \delta_{\varepsilon}\left(E-\frac{1}{2}\left(E_{n}+E_{m}\right)\right) \delta_{\varepsilon} \cdot\left(\Delta E-\left(E_{n}-E_{m}\right)\right)
$$

where $\delta_{\varepsilon}(x)$ is a Dirac delta function smeared over a range $\varepsilon$

$$
\delta_{\varepsilon}(x)=\frac{1}{\sqrt{2 \pi \varepsilon}} \mathrm{e}^{-x^{2} / 2 \varepsilon^{2}} .
$$

The sum $S(E, \Delta E)$ can be related to the classical correlation function of $A(q, p)$ under the motion generated by the Hamiltonian $H(\boldsymbol{q}, \boldsymbol{p})$ (Wilkinson 1987b). This correlation function is defined by

$$
C_{A}(E, t)=\int \mathrm{d} \alpha A(\alpha) A\left(\alpha_{t}\right) \delta(E-H(\alpha))
$$

where $\alpha$ is a phase space point $(\boldsymbol{q}, \boldsymbol{p})$, and $\alpha_{t}$ is the point obtained by evolving $\alpha$ for time $t$ under the Hamiltonian $H(\alpha)$. Provided that $\varepsilon$ and $\varepsilon^{\prime}$ are much greater than $h / \tau_{1}$, where $\tau_{1}$ is the period of the shortest periodic orbit of $H(\alpha)$, the sum $S(E, \Delta E)$ is given by

$$
S(E, \Delta E)=\frac{1}{(2 \pi \hbar)^{d+1}} \int_{-\infty}^{\infty} \mathrm{d} t \mathrm{e}^{\mathrm{i} \Delta E t / h} C_{A}(E, t) .
$$

When $\varepsilon \leqslant h / \tau_{1}$, there are corrections to (3.4) which depend on properties of the periodic orbits (Wilkinson 1987b). These corrections are of higher order in $\hbar$, and do not have any significant effect on the spectral statistics in the semiclassical limit. The variance of the matrix elements is given by

$$
\sigma^{2}(E, \Delta E)=\frac{S(E, \Delta E)}{(n(E))^{2}}
$$

where $n(E)$ is the smoothed density of states. 
Typically, the perturbation $A(\alpha)$ is a rapidly varying function, so that its correlation function will decay to zero in a very short time $\tau_{0}$, independent of the dynamics of $H(\alpha)$. Because $\sigma^{2}$ depends on the Fourier transform of this correlation function (3.4), the variance $\sigma^{2}$ is independent of the energy difference $\Delta E$, for $\Delta E$ less than some large value $h / \tau_{0}$. This is consistent with the arguments of $\S 2$, showing that the matrix elements are distributed in accordance with random matrix theory.

It will be useful to define the variance of $A,\left\langle A^{2}\right\rangle$, and to define the decorrelation time $\tau_{0}$ more precisely. These quantities are defined through the formulae

$$
\begin{aligned}
& C_{A}(E, 0)=\frac{\mathrm{d} V}{\mathrm{~d} E}\left\langle A^{2}\right\rangle \\
& \int_{-\infty}^{\infty} \mathrm{d} t C_{A}(E, t)=\frac{\mathrm{d} V}{\mathrm{~d} E}\left\langle A^{2}\right\rangle \tau_{0}
\end{aligned}
$$

where $(\mathrm{d} V / \mathrm{d} E) \delta E$ is the volume of an energy shell in phase space of thickness $\delta E$, i.e.

$$
\frac{\mathrm{d} V}{\mathrm{~d} E}=\int \mathrm{d} \alpha \delta(E-H(\alpha)) .
$$

Using (3.4), (3.5) and the above definitions, we can write

$$
\sigma^{2}=\frac{\left\langle A^{2}\right\rangle \tau_{0}}{2 \pi \hbar n_{0}}
$$

where we have made use of the Weyl approximation (Berry 1983) for the smoothed density of states

$$
n_{0}(E)=\frac{1}{(2 \pi \hbar)^{d}} \frac{\mathrm{d} V}{\mathrm{~d} E} .
$$

\subsection{Diagonal matrix elements}

Now consider the sum of diagonal matrix elements

$$
f(E)=\sum_{n} A_{n n} \delta_{\varepsilon}\left(E-E_{n}\right)
$$

where $\delta_{\varepsilon}(x)$ has the same meaning as in (3.2). This sum is an average of $A_{n n}$ over a small range of energy, of length $\varepsilon$. An expression will be derived for $f(E)$ in the form of a sum over periodic orbits. This expression is analogous to Gutzwiller's formula for the density of states (Gutzwiller 1967) and is equivalent to it if we set $\hat{A}=\hat{I}$ (the identity operator) when $f(E)=n(E)$ (the smoothed density of states).

The sum $f(E)$ can be written

$$
f(E)=\frac{1}{2 \pi \hbar} \int_{-\infty}^{\infty} \mathrm{d} t \exp (\mathrm{i} E t / \hbar) \exp \left(-\varepsilon^{2} t^{2} / 2 h^{2}\right) \operatorname{Tr}(\hat{U}(t) \hat{A})
$$

where $\hat{U}(t)=\exp (-\mathrm{i} \hat{H} t / \hbar)$ is the evolution operator. It is helpful to consider the expression for the trace in (3.12) in a basis of coherent states $|\alpha\rangle$

$$
\begin{aligned}
\operatorname{Tr}(\hat{U}(t) \hat{A}) & =\frac{1}{(2 \pi \hbar)^{d}} \int \mathrm{d} \alpha\langle\alpha|\hat{U}(t) \hat{A}| \alpha\rangle \\
& =\frac{1}{(2 \pi \hbar)^{d}} \int \mathrm{d} \alpha A(\alpha)\langle\alpha|\hat{U}(t)| \alpha\rangle
\end{aligned}
$$


where the second equality holds in the semiclassical limit, $\hbar \rightarrow 0$. The matrix element $\langle\alpha|\hat{U}(t)| \alpha\rangle$ is very small except when the classical dynamics maps $\alpha$ into a nearby point after time $t$. The function $f(E)$ can therefore be written as a sum over periodic trajectories in phase space

$$
f(E)=f_{0}(E)+\sum_{j} f_{j}(E) \exp \left(-\varepsilon^{2} \tau_{j}^{2} / 2 \hbar^{2}\right)
$$

where the first term in the summation comes from contributions to (3.13) with $t \simeq 0$ and the $j$ th term comes from the $j$ th periodic orbit, with period $\tau_{j}(E)$. These periodic orbit contributions can be evaluated by standard methods (Berry 1983, Wilkinson $1987 \mathrm{~b})$. The term $f_{0}(E)$ is the average of $A(\alpha)$ over the energy shell

$$
f_{0}(E)=\frac{1}{(2 \pi \hbar)^{d}} \int \mathrm{d} \alpha A(\alpha) \delta(E-H(\alpha))=n_{0}(E) \bar{A}_{0}
$$

where the second equality defines $\bar{A}_{0}$. The periodic orbit terms are

$$
\begin{aligned}
f_{j}(E) & =\alpha_{j}(E) \exp \left(\mathrm{i} \sigma_{j}(E) / \hbar\right) \frac{1}{\tau_{j}} \int_{0}^{\tau_{j}} \mathrm{~d} \tau A_{j}(\tau ; E) \\
& =\alpha_{j} \bar{A}_{j} \exp \left(\mathrm{i} \sigma_{j} / \hbar\right)
\end{aligned}
$$

where $\sigma_{j}(E)$ is the classical action of the $j$ th orbit, plus a correction term for the number of focal points, $\gamma_{j}$,

$$
\sigma_{j}(E)=\oint_{j} \boldsymbol{p} \mathrm{d} \boldsymbol{q}+\frac{1}{2} \pi \gamma_{j} \hbar
$$

and $\bar{A}_{j}(E)$ is the time-weighted average of $A(\alpha)$ evaluated on the $j$ th orbit at energy $E$. The amplitudes $\alpha_{j}(E)$ depend on the stability of the periodic orbits and will not be discussed here; they can be determined by setting $\bar{A}_{j}=1$ and comparing with standard expressions for the density of states, $n(E)$, reviewed by Berry (1983).

Combining equations (3.14)-(3.17), we have

$$
f(E)=\bar{A}_{0} n_{0}+\sum_{j} \alpha_{j} \bar{A}_{j} \exp \left(i \sigma_{j} / \hbar\right) \exp \left(-\varepsilon^{2} \tau_{j}^{2} / 2 \hbar^{2}\right)
$$

The sum over periodic orbits $j$ includes orbits traced backwards in time, for which $\sigma_{-j}=-\sigma_{j}$, so that the sum is real. Using the result

$$
\left.\frac{\partial \sigma}{\partial E}\right|_{E}=\tau_{j}(E)
$$

we see that the periodic orbit corrections oscillate as a function of energy, with period $h / \tau_{j}$. In the neighbourhood of the energy $E_{0}$ we can therefore write (3.19) as a sum of periodic terms

$f(E)=\bar{A}_{0} n_{0}\left(E_{0}\right)+\sum_{j} \alpha_{j}\left(E_{0}\right) \bar{A}_{j}\left(E_{0}\right) \exp \left(\mathrm{i} \sigma_{j}\left(E_{0}\right) / \hbar\right) \exp \left(-\varepsilon^{2} \tau_{j}^{2} / \hbar\right) \exp \left(\mathrm{i}\left(E-E_{0}\right) \tau_{j} / \hbar\right)$.

This expression is in the form of a Fourier expansion for $f(E)$. The Fourier transform $\tilde{f}(\tau)$ of $f(E)$ has a discrete spectrum, with frequencies proportional to $\tau_{j}$. The amplitudes are the product of four terms: an amplitude $\alpha_{j}$ which characterises the stability of the perodic orbit, the time-weighted average $\bar{A}_{j}$ of $A$ over the periodic orbit, a complex phase factor and a term which suppresses contributions from orbits with periods greater than $h / \varepsilon$. 
It may be helpful to contrast the treatment of the sum rules for the diagonal and off-diagonal matrix elements. In both cases, there is an average term, plus small oscillatory corrections related to periodic classical orbits. In the case of the off-diagonal matrix elements the small periodic orbit corrections can be ignored. In the case of the diagonal sum rule, the average term describes a uniform shift of all the energy levels, without changing their relative positions, and the smaller periodic orbit terms are of crucial importance. For this reason, the periodic orbit terms were evaluated in this section, but not in $\$ 3.1$.

The periodic orbit sum (3.20) is only valid for values of the energy resolution $\varepsilon$ greater than some minimum value $\varepsilon^{*}(\hbar)$. The reason is that the semiclassical approximations used to derive the periodic orbit corrections are not valid in the limit $\tau_{j} \rightarrow \infty$ with $\hbar$ fixed. If the periodic orbits are unstable, the semiclassical approximation is expected to break down for times greater than

$$
\tau^{*} \sim 1 / \gamma \ln \left(S_{0} / \hbar\right)
$$

where $\gamma$ is the Lyapunov exponent of the orbit and $S_{0}$ is some characteristic classical action of the system (Wilkinson 1987b). The corresponding minimum value of $\varepsilon$ is therefore

$$
\varepsilon^{*}=\hbar / \tau^{*}=\hbar \gamma / \ln \left(S_{0} / \hbar\right)
$$

\subsection{A sum rule for the spectral intensities}

The sum $f(E)$, which is the mean value of $A_{n n}$ averaged over a short range of energy of length $o(\varepsilon)$, has been evaluated semiclassically in terms of a sum over periodic orbits. We can also attempt to calculate $f(E)$ using random matrix theory: the matrix elements $A_{n n}$ are predicted to be uncorrelated random variables, with variance $\beta \sigma^{2}$. The sum $f(E)$ is therefore a random variable, with variance

$$
\begin{aligned}
\left\langle\left(f-f_{0}\right)^{2}\right\rangle & =\sum_{n}\left\langle\left(A_{n n}-f_{0}\right)^{2}\right\rangle \delta_{\varepsilon}^{2}\left(E-E_{n}\right) \\
& =\beta \sigma^{2} n_{0} \int_{-\infty}^{\infty} \mathrm{d} x \frac{1}{2 \pi \varepsilon^{2}} \exp \left(-x^{2} / \varepsilon^{2}\right) \\
& =\frac{\sqrt{\pi}}{2 \pi} \frac{\beta \sigma^{2} n_{0}}{\varepsilon}
\end{aligned}
$$

provided $\varepsilon n_{0} \gg 1$, so that the sum can be approximated by an integral. This result must be compared with a calculation of the variance from the periodic orbit sum (3.20). It will be found that the two expressions agree when $\varepsilon \ll h / \tau_{1}$, where $\tau_{1}$ is the period of the shortest closed orbit, but that for $\varepsilon \geqslant h / \tau_{1}$ the results diverge. This shows that the diagonal matrix elements are indeed locally uncorrelated, but that they are collectively constrained by the sum rule $(3.20)$.

From (3.20), the variance of $f$ is given by

$$
\begin{aligned}
\left\langle\left(f-f_{0}\right)^{2}\right\rangle=\sum_{i j} & \alpha_{i} \alpha_{j}\left\langle\bar{A}_{i} \bar{A}_{j}\right\rangle \exp \left(\left.\frac{\mathrm{i}\left(\sigma_{i}+\sigma_{j}\right)}{\hbar}\right|_{E_{0}}\right) \exp \left(-\frac{\varepsilon^{2}\left(\tau_{1}^{2}+\tau_{j}^{2}\right)}{2 \hbar^{2}}\right) \\
& \times \exp \left[(\mathrm{i} / \hbar)\left(E-E_{0}\right)\left(\tau_{i}+\tau_{j}\right)\right] .
\end{aligned}
$$

Terms corresponding to inequivalent orbits disappear from the sum, since in this case $\left\langle\bar{A}_{i} \bar{A}_{j}\right\rangle=0$. The remaining terms are of two types. Terms combining an orbit of period 
$\tau_{i}$ with its reverse traced orbit, period $-\tau_{i}$, give energy-independent contributions. Terms in which an orbit is paired with itself give contributions which oscillate as a function of energy; when many orbits are included in the sum these osciliatory terms combine incoherently, and give a negligible contribution. Considering only the energyindependent terms, we have

$$
\left\langle\left(f-f_{0}\right)^{2}\right\rangle=\beta \sum_{j} \alpha_{j}^{2}\left\langle\bar{A}_{j}^{2}\right\rangle \exp \left(-\varepsilon^{2} \tau_{j}^{2} / \hbar^{2}\right)
$$

where the sum is now confined to positive time traversals of the periodic orbits. The factor $\beta$ multiplying the sum is required because, when there is time reversal symmetry, orbits occur as equivalent pairs which doubles the number of terms for which $\left\langle\overline{A_{i}} \bar{A}_{j}\right\rangle$ is non-zero.

The sum (3.25) must now be compared with the expression (3.23) derived from random matrix theory. The two expressions are clearly different when $\varepsilon \gg h / \tau_{1}$, since in this case (3.25) is exponentially small. When $n_{0}^{-1} \ll \varepsilon \ll h / \tau_{1}$, many periodic orbits contribute to the sum in (3.25), and we require some information about the size of the amplitudes $\alpha_{j}$ for long orbits. The necessary information is contained in a sum rule derived by Hannay and Ozorio de Almeida (1984). The sum rule states that, if $\alpha_{j}$ are the amplitudes corresponding to unstable periodic orbits

$$
s(\tau)=\lim _{\tau \rightarrow \infty} \sum_{\substack{j \\ \tau_{j}<\tau}} \alpha_{j}^{2}=\frac{\tau^{2}}{(2 \pi \hbar)^{2}} .
$$

It follows that for small $\varepsilon$, the sum (3.25) can be approximated by the integral

$$
\begin{aligned}
\left\langle\left(f-f_{0}\right)\right\rangle^{2} & =\beta \int_{0}^{\infty} \mathrm{d} \tau \frac{\mathrm{d} s}{\mathrm{~d} \tau}\left\langle\bar{A}_{j}^{2}\right\rangle \exp \left(-\varepsilon^{2} \tau^{2} / \hbar^{2}\right) \\
& =\frac{2 \beta}{(2 \pi \hbar)^{2}} \int_{0}^{\infty} \mathrm{d} \tau \tau\left\langle\bar{A}_{j}^{2}\right\rangle \exp \left(-\varepsilon^{2} \tau^{2} / \hbar^{2}\right) .
\end{aligned}
$$

The variance of $A_{j}$ is (using 3.7)

$$
\begin{aligned}
\left\langle\bar{A}_{j}^{2}\right\rangle & =\frac{1}{\tau_{j}^{2}} \int_{0}^{\tau_{j}} \mathrm{~d} \tau \int_{0}^{\tau_{j}} \mathrm{~d} \tau^{\prime}\left\langle A_{j}(\tau) A_{j}\left(\tau^{\prime}\right)\right\rangle \\
& =\frac{1}{\tau_{j}^{2}} \int_{0}^{\tau_{j}} \mathrm{~d} \tau \int_{-\tau}^{\tau_{j}-\tau} \mathrm{d} \tau^{\prime}\left(A_{j}(\tau) A_{j}\left(\tau+\tau^{\prime}\right)\right\rangle \\
& =\left\langle A^{2}\right\rangle \tau_{0} / \tau_{j}
\end{aligned}
$$

where the last equality uses an ergodic property, which only applies to long closed orbits. Substituting (3.28) into (3.27) and using (3.9), we find

$$
\left\langle\left(f-f_{0}\right)^{2}\right\rangle=\frac{2\left\langle A^{2}\right\rangle \tau_{0} \beta}{(2 \pi \hbar)^{2}} \int_{0}^{\infty} \mathrm{d} \tau \exp \left(-\varepsilon^{2} \tau^{2} / \hbar^{2}\right)=\frac{\sqrt{\pi}}{2 \pi} \frac{\beta \sigma^{2} n_{0}}{\varepsilon}
$$

for small $\varepsilon$. This is in agreement with the prediction of random matrix theory (3.23).

\section{Discussion}

The results of $\S 2$ showed that the effect of the ambiguity of quantisation is equivalent to considering the energy levels as mutually repelling particles embedded in a viscous 
medium (equations (2.10) and (2.12)), driven by an uncorrelated Brownian motion (equation (2.11)). Section 3 considered in detail the consequences of the fact that the perturbation $\hat{A}$ has an analytic classical limit $A(q, p)$. This leads to the important conclusion that, although the diagonal matrix elements representing the Brownian motion are locally uncorrelated, they satisfy the sum rule (3.20). In the limit $\varepsilon \rightarrow 0$, the sum $f(E)$ describes the spatial distribution of the random walk which drives the motion of the energy level gas. The sum rule (3.20) shows that the spectrum $\tilde{f}(\tau)$ of this random walk only contains a discrete set of frequencies

$$
\begin{aligned}
& f(E)=\int \mathrm{d} \tau \tilde{f}(\tau) \exp \left[\mathrm{i}\left(E-E_{0}\right) \tau / \hbar\right] \\
& \tilde{f}(\tau)=\sum_{j} \alpha_{j} \bar{A}_{j} \exp \left[(1 / \hbar) \sigma_{j}\left(E_{0}\right)\right] \delta\left(\tau-\tau_{j}\right) .
\end{aligned}
$$

This should be contrasted with the uniform spectral intensity which would characterise a totally uncorrelated distribution (see figure 1 ). The results of $\S 3.3$ show that the spectral intensity agrees with the uncorrelated distribution for large values of $\tau$.

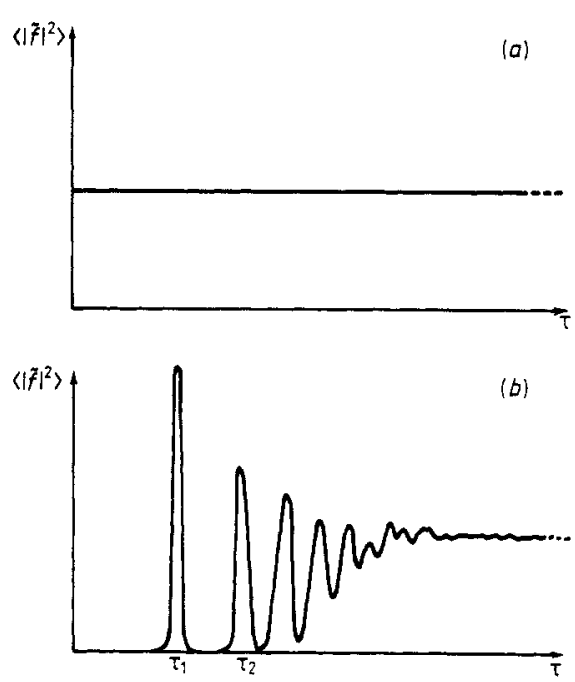

Figure 1. (a). In random matrix theory, the diagonal matrix elements are completely uncorrelated, so that $f(E)=\sum_{n} A_{n n} \delta\left(E-E_{n}\right)$ resembles white noise, which has a uniform spectral density, $\left\langle\left.\tilde{f}(\tau)\right|^{2}\right\rangle$. (b) In the case of a system with a chaotic classical limit, $\tilde{f}(\tau)$ is a sum of contributions from classical periodic orbits, with periods $\tau_{j}$. The contributions from the long orbits sum to the same spectral density as random matrix theory at large $\tau$.

Now consider the implications of the ambiguity of quantisation for the spectral statistics. Initially, the Hamiltonian is quantised so that it is represented by an operator $\hat{H}_{0}$. The smoothed density of states is given by the periodic orbit sum (Gutzwiller 1967)

$$
\begin{aligned}
n(E) & =\sum_{n} \delta_{\varepsilon}\left(E-E_{n}\right) \\
& =\sum_{j} \alpha_{j} \exp \left[(\mathrm{i} / \hbar) \sigma_{j}\left(E_{0}\right)\right] \exp \left[\mathrm{i}\left(E-E_{0}\right) \tau_{j} / \hbar\right] \exp \left(-\varepsilon^{2} \tau_{j}^{2} / 2 \hbar^{2}\right)
\end{aligned}
$$


where the amplitudes $\alpha_{j}$ are the same as those occurring in (3.20), and are determined to lowest order by the stability properties of the classical periodic orbits. The effect of the perturbation $\hat{A}$ can be analysed in terms of its effect on the amplitudes $\alpha_{j}$. This is done in appendix $2:$ it is shown that, provided the wavelength $h / \tau_{j}$ of the fluctuation is much greater than the mean level separation, a continuum approximation is valid and that the amplitudes $\alpha_{j}$ evolve independently. The perturbation $\hat{A}$ causes $\alpha_{j}$ to undergo Brownian motion with a diffusion constant proportional to $\alpha_{j}^{2}$

$$
\left\langle\delta \alpha_{j}^{2}\right\rangle=D_{j}^{\prime} \delta \Gamma=\frac{2 \pi \kappa n_{0}}{\hbar} \tau_{j} \alpha_{j}^{2} \delta \Gamma
$$

and the motion of the coordinate $\alpha$, is restrained by a linear restoring force

$$
\left\langle\delta \alpha_{j}\right\rangle=-\frac{\pi \kappa n_{0} \tau_{j}}{\hbar} \alpha_{j} \delta \Gamma
$$

Equation (4.4) shows that the characteristic response time for the $j$ th mode is

$$
\Gamma_{j}=\frac{\hbar}{\pi \kappa n_{0} \tau_{j}}
$$

Note that this is longer for the long-wavelength modes (which have smaller classical perods, $\tau_{j}$ ).

The perturbation $\hat{A}$ is very small, of size $o\left(\hbar^{2}\right)$. This implies that the random walk should only be allowed to proceed for a short time $\Gamma^{*}$. Even though the perturbation is very small, it can be large enough to shift the energy levels by many times their mean spacing, in which case

$$
\Lambda=\sigma^{2} n_{0}^{2} \gg 1 \text {. }
$$

Using (2.6), the time increment $\Gamma^{*}$ is given by

$$
\Gamma^{*}=\Lambda / \kappa n_{0}^{2} \text {. }
$$

If $\Gamma^{*} / \Gamma_{j} \gg 1$, then the mode is able to come into thermodynamic equilibrium, whereas if $\Gamma^{*} / \Gamma_{j} \ll 1$ the mode is not significantly affected by the Brownian motion. Comparing (4.5) and (4.7) we find

$$
\Gamma^{*} / \Gamma_{j}=\left(\pi \Lambda / n_{0} \hbar\right) \tau_{j}
$$

Since $\Lambda=o\left(\hbar^{2-d}\right)$, and $n_{0}=o\left(\hbar^{-d}\right)$, this ratio is of order unity when $\tau_{j}=o\left(\hbar^{-1}\right)$. This result shows that the long-wavelength modes are not affected by the ambiguity of quantisation, but the short-wavelength modes are brought into thermodynamic equilibrium. The results of random matrix theory are therefore valid for short ranges of energy, but not for long-ranged properties of the spectrum.

\section{The effect of symmetries}

In $\S 2$ it was assumed that the perturbation $\hat{A}$ does not respect any of the symmetries of the Hamiltonian $\hat{H}_{0}$, except time reversal symmetry (if present). This section will clarify the meaning of this assumption, and show that it is justified for chaotic systems but not in the case of integrable or quasi-integrable systems.

If $\hat{H}_{0}$ has a symmetry, or constant of motion, represented by an operator $\hat{K}$, then

$$
\left[\hat{H}_{0}, \hat{\mathbf{K}}\right]=0 \text {. }
$$


The perturbation $\hat{A}$ respects this symmetry if there is a corresponding perturbation $\hat{B}$ of $\hat{K}$, such that $\hat{K}$ remains a constant of motion:

$$
\left[\hat{H}_{0}+\hat{A}, \hat{K}+\hat{B}\right]=0
$$

of if $\hat{A}$ is small

$$
[\hat{A}, \hat{K}]+\left[\hat{H}_{0}, \hat{B}\right]=0 .
$$

Taking matrix elements of (5.3), we find

$$
A_{n m} K_{m}-A_{m n} K_{n}=B_{n m} E_{m}-B_{m n} E_{n} \text {. }
$$

This result implies that when $K_{n} \neq K_{m}, A_{n m} \rightarrow 0$ as $E_{n}-E_{m} \rightarrow 0$, in which case the energy levels $E_{n}, E_{m}$ do not show level repulsion. The model derived in $\S 2$ is therefore not valid when $\hat{A}$ respects a symmetry of $\hat{H}_{0}$ (except time reversal symmetry which is not of the type represented by (5.1), in which there is a constant of motion).

If the classical Hamiltonian corresponding to $\hat{H}_{0}$ is chaotic, then for almost all perturbations $\hat{A}$ will not respect any symmetry of $\hat{H}_{0}$. This is because the only constraint on $\hat{A}$ is that it has an analytic classical limit, so that the only symmetry of $\hat{H}_{0}$ which $\hat{A}$ can respect is one which corresponds to a classical constant of motion $K(q, p)$. By definition, a chaotic Hamiltonian has no such constant of motion.

If the classical Hamiltonian does have a constant of motion $K(\boldsymbol{q}, \boldsymbol{p})$, then there exist corresponding operators $\hat{K}$ which are approximate constants of motion; from (A1.5) we see that $\hat{K}$ can always satisfy

$$
\left[\hat{H}_{0}, \hat{K}\right]=o\left(\hbar^{2}\right)
$$

but it is not usually possible to find an operator $\hat{K}$ such that $\left[\hat{H}_{0}, \hat{K}\right]=0$ exactly (Hietarinta 1984). Equation (5.5) suggests that when a perturbation is applied which respects this symmetry, energy levels will be repelled when they have a separation $\Delta E \sim \mathrm{o}\left(\hbar^{2}\right)$. For systems with $d$ degrees of freedom, the mean separation of energy is $o\left(\hbar^{d}\right)$, so that when $d \geqslant 2$, equation (5.5) does not in itself imply a breakdown of energy-level repulsion.

If the constant of motion is such that the classical motion is confined to tori in phase space (as is the case for integrable or quasi-integrable systems) then the situation is rather more complex. There are two important points. First, if a perturbation is applied which respects this constant of motion, then numerical experiments (Wilkinson 1987a) have shown that energy levels can approach to a minimum separation much smaller than the mean level separation. Theoretical arguments (Wilkinson 1986) show that in quasi-integrable systems this minimum separation is

$$
\Delta E=C \mathrm{e}^{-S / \hbar}
$$

where $S$ is a constant and $C$ has a power law dependence on $\hbar$, and that for exactly integrable systems $\Delta E$ can vanish in an even more singular manner as $\hbar \rightarrow 0$. The second important point is that, although the only constraint on the perturbation $\hat{A}$ is that it has an analytic classical limit, the KAM theorem shows that all perturbations in this class preserve the constant of motion. Integrable or quasi-integrable systems do not therefore satisfy the requirement that the perturbation $\hat{A}$ should not respect any symmetry of $\hat{H}_{0}$.

Systems which have a constant of motion, but where the motion does not occupy a torus in phase space are called pseudo-integrable (Richens and Berry 1981). Most rational-angled triangular billiards are examples of this type of motion. Unlike torus quantised systems, there does not appear to be any theoretical reason to expect the range of level repulsion to be less than $o\left(\hbar^{2}\right)$, as predicted by (5.5). Numerical results 
show a level spacing distribution similar to the Wigner distribution (Richens and Berry 1981, Berry and Wilkinson 1984), in agreement with this prediction. Long-ranged spectral statistics are, however, expected to show deviations from the predictions of random matrix theory at much smaller energy ranges than chaotic systems. The reason is that the sum rule derived in $\S 3.3$ is only valid for systems with unstable periodic orbits. If the orbits are unstable, this sum rule shows that the random matrix model becomes correct for energy ranges a few times smaller than $h / \tau_{1}$, where $\tau_{1}$ is the period of the shortest periodic orbit. In pseudo-integrable systems, the periodic orbits are marginally stable and non-isolated, and in this case (3.26) is not valid, and the random matrix behaviour must be restricted to much smaller energy scales, where the terms in the periodic orbit sum corresponding to very long orbits are not meaningful.

\section{Summary and conclusion}

Random matrix theory suggests that any quantum mechanical system which has a large number of energy levels should have one of three universal types of spectral statistics, provided that it has no constants of motion (Porter 1965). These universality classes are the Gaussian orthogonal and Gaussian unitary ensembles, which have already been mentioned, and the less usual Gaussian symplectic ensemble (Dyson 1962a). Semiclassical systems with a chaotic classical limit have no obvious constants of motion (provided trivial geometrical symmetries are removed), and it might be expected that their spectra should have the properties of one of these ensembles. This is, however, at variance with the results of Gutzwiller (1967) and others, which show that the fluctuations in the density of states contain only a discrete spectrum of frequencies, corresponding to the periods of classical periodic orbits, instead of the continuous spectrum predicted by standard random matrix models.

This paper has presented an analysis of this problem, which is based on the fact that the ambiguity of quantisation $H(\boldsymbol{q}, \boldsymbol{p}) \rightarrow \hat{H}$ can be represented by a perturbation $\hat{A}$ added to $\hat{H}$. This perturbation does not appear to satisfy any constraint, other than that it should have a classical limit $A(q, p)$. Applying this perturbation will therefore destroy any constants of motion which do not have a classical limit, so that only classical constants of motion can be preserved. If the system has a chaotic classical limit, there are no such constants of motion. If the system is integrable or quasiintegrable however, the KAM theorem shows that the perturbation does not destroy the constant of motion. Random matrix theory should therefore be applicable to classically chaotic systems but not to integrable or quasi-integrable ones.

Following the work of Dyson (1962b), the effect of the perturbation $\hat{A}$ can be analysed in a physically transparent way by making the perturbation execute a random walk in function space. The shift of the energy levels is calculated using perturbation theory; because $\hat{A}$ undergoes a random walk both first-order and second-order terms are equally significant. These terms can be interpreted by regarding the energy levels $E_{n}$ as the positions of particles embedded in a viscous medium. The first-order perturbation represents diffusive motion, and the second-order term represents a repulsive force between particles.

The fact that the perturbation $\hat{A}$ has a classical limit has important consequences. Although the diagonal matrix elements $A_{n n}$ are locally uncorrelated, they satisfy the sum rule (3.20), which shows that the diffusive motion is driven by a discrete spectrum of frequencies, corresponding to the periods of classical periodic orbits. The classical 
sum rule of Hannay and Ozorio de Almeida (1984) can be used to verify that if the periodic orbits are unstable, the diagonal matrix elements are locally uncorrelated.

The configuration of the energy level gas can be Fourier analysed; the density fluctuations have a discrete spectrum, with frequencies equal to $\tau_{j} / h$, where $\tau_{j}$ is the period of the $j$ th classical periodic orbit. The random perturbation $\hat{A}$ causes the amplitudes $\alpha_{j}$ of these density fluctuations to undergo diffusive motion. This diffusive motion is characterised by a time constant $\Gamma_{j}$, which is inversely proportional to the period $\tau_{j}$. Since the perturbation $\hat{A}$ is small, of size $o\left(\hbar^{2}\right)$, the diffusive motion does not act for long enough to alter the amplitudes of the long-wavelength fluctuations, which have a long relaxation time. The short-wavelength fluctuations, corresponding to long periods $\tau_{j}$, have a short relaxation time, and their amplitudes $\alpha_{j}$ are strongly affected by the diffusive motion, and come into thermodynamic equilibrium. Shortranged spectral statistics therefore correspond to the predictions of random matrix theory, whilst long-ranged fluctuations are non-universal and their amplitudes $\alpha_{j}$ can be calculated explicitly.

Pseudo-integrable systems are still not completely understood. Although they have a classical constant of motion, the corresponding quantum operator is not an exact constant of motion, and energy level repulsion is not suppressed. These systems do not have unstable periodic orbits, however, and the sum rule of Hannay and Ozorio de Almeida does not predict a correspondence with random matrix theory. It seems the random matrix picture is only correct for very short energy ranges, where the periodic orbit corrections are not meaningful.

\section{Acknowledgment}

I am grateful to the Nuffield Foundation for the award of a research grant. This work was not supported by any military agency.

\section{Appendix 1.}

There is in general no unique way of assigning a Hermitian operator $\hat{H}$ to a classical Hamiltonian function $H(\boldsymbol{q}, \boldsymbol{p})$. It will be shown that this ambiguity can be represented by a small perturbation of size $o\left(\hbar^{2}\right)$.

There is more than one way of approaching this problem. Let us fix the correspondence between classical functions and quantum operators by using the Weyl quantisation to go from $A(q, p)$ to the operator $\hat{A}$. The Weyl quantisation (Weyl 1927, Groenewold 1946) will be described below. We then consider the effect of applying a classical canonical transformation to $A(q, p)$. If we quantise the transformed function, $A^{\prime}(q, p)$, using the Weyl rule, we obtain an operator $A^{\prime}$ which is not unitarily equivalent to $A$ but which differs from it by a term of size $o\left(\hbar^{2}\right)$.

The Weyl quantisation is given by

$$
\hat{A}=\frac{1}{(2 \pi \hbar)^{d}} \iint \mathrm{d}^{d} Q \mathrm{~d}^{d} P a(\boldsymbol{Q}, \boldsymbol{P}) \exp [(-\mathrm{i} / \hbar)(\boldsymbol{Q} \cdot \hat{\boldsymbol{q}}+\boldsymbol{P} \cdot \hat{\boldsymbol{p}})]
$$

where $a(\boldsymbol{Q}, \boldsymbol{P})$ is the Fourier transform of $\boldsymbol{A}(\boldsymbol{q}, \boldsymbol{p})$

$$
a(\boldsymbol{Q}, \boldsymbol{P})=\frac{1}{(2 \pi \hbar)^{d}} \iint \mathrm{d}^{d} q \mathrm{~d}^{d} p \boldsymbol{A}(\boldsymbol{q}, \boldsymbol{p}) \exp [(\mathrm{i} / \hbar)(\boldsymbol{Q} \cdot \boldsymbol{q}+\boldsymbol{P} \cdot \boldsymbol{p})]
$$


Consider the effect of applying an infinitesimal canonical transformation of $A(q, p)$, generated by a Hamiltonian $G(\boldsymbol{q}, \boldsymbol{p})$, acting for a time $\delta t$

$$
A^{\prime}(\boldsymbol{q}, \boldsymbol{p})=\boldsymbol{A}(\boldsymbol{q}, \boldsymbol{p})+\{A, G\} \delta t+o\left(\delta t^{2}\right)
$$

where $\{A, B\}$ is the Poisson bracket of $A$ and $B$. The corresponding quantum mechanical transformation is a unitary transformation generated by $\hat{G}$

$$
\begin{aligned}
\hat{A}^{\prime \prime} & =\mathrm{e}^{\mathrm{i} \hat{G} \delta t / \hbar} \hat{A} \mathrm{e}^{-\mathrm{i} \hat{G} \delta t / \hbar} \\
& =\hat{A}+(\mathrm{i} / \hbar)[\hat{A}, \hat{G}] \delta t+\mathrm{o}\left(\delta t^{2}\right)
\end{aligned}
$$

where $\hat{G}$ is obtained from $\hat{G}(\boldsymbol{q}, \boldsymbol{p})$ using the Weyl quantisation. Compare $\hat{A}^{\prime \prime}$ with the operator $\hat{A}^{\prime}$ obtained by quantising $A^{\prime}(q, p)$. The two are only equal if $(i / \hbar)[A, B]=$ $\{A, B\}$ in the Weyl quantisation. In general this is not true: Moyal (1949) showed that, if $\hat{C}=(i / \hbar)[\hat{A}, \hat{B}]$, the corresponding classical function is

$$
\begin{aligned}
C(\boldsymbol{q}, \boldsymbol{p})=\frac{2}{\hbar} \sin & \left.\left\{\frac{\hbar}{2}\left[\frac{\partial}{\partial \boldsymbol{q}_{1}} \frac{\partial}{\partial \boldsymbol{p}_{2}}-\frac{\partial}{\partial \boldsymbol{p}_{1}} \frac{\partial}{\partial \boldsymbol{q}_{2}}\right] A\left(\boldsymbol{q}_{1}, \boldsymbol{p}_{1}\right) B\left(\boldsymbol{q}_{2}, \boldsymbol{p}_{2}\right)\right\}\right|_{\substack{\boldsymbol{q}_{1}=q_{2}=\boldsymbol{q} \\
\boldsymbol{p}_{1}=\mathbf{p}_{2}=\mathbf{p}}} \\
= & \{A, B\}+o\left(\hbar^{2}\right) .
\end{aligned}
$$

In general, therefore, $\hat{A}^{\prime}$ and $\hat{A}^{\prime \prime}$ differ by $o\left(\hbar^{2}\right)$. If $H$ is the Hamiltonian function of a given classical system, we have seen that the results of applying the Weyl quantisation depend on the choice of phase space coordinates, and the operators $\hat{H}$ differ by $o\left(\hbar^{2}\right)$. This perturbation representing the ambiguity of quantisation is arbitrary, apart from the constraint that it should have a classical limit.

If the Hamiltonian has time reversal symmetry, it is appropriate to impose another constraint on the perturbation. A classical Hamiltonian has time reversal symmetry if there exist coordinates $(\boldsymbol{q}, \boldsymbol{p})$ in which $H(\boldsymbol{q}, \boldsymbol{p})=H(\boldsymbol{q},-\boldsymbol{p})$. If the Hamiltonian is quantised by applying the Weyl rule in these coordinates, the resulting quantum Hamiltonian $\hat{H}$ also has time reversal symmetry, and this is usually the physically correct quantisation. In some coordinates, however, the Weyl rule quantisation will not have exact time reversal symmetry, and this alters the spectral statistics. Since we are only interested in physically reasonable quantisations, which have time reversal symmetry, the perturbation should be constrained to preserve this symmetry.

\section{Appendix 2}

The motion of the energy level gas can be Fourier analysed, and the amplitudes of the modes used as dynamical variables. For small amplitude disturbances modes with wavelengths large compared with the mean level separation behave as independent harmonic oscillators, driven by a random force. This appendix will analyse the motion of these long-wavelength modes in detail.

Let $E_{n}^{0}$ be the position of the $n$th energy level which would be predicted by applying the Weyl rule $(3.10)$ for the density of states, without any periodic orbit corrections. The displacement of the $n$th energy level

$$
X\left(E_{n}\right)=E_{n}-E_{n}^{0}
$$

is related to the corrections $\Delta n$ to the density of states by

$$
\Delta n(E)=n(E)-n_{0}(E)=-n_{0}(E) \mathrm{d} X(E) / \mathrm{d} E .
$$


The displacement can be Fourier analysed

$$
X(E)=\int \mathrm{d} \tau \tilde{X}(\tau) \exp (\mathrm{i} E \tau / \hbar)
$$

For small amplitude corrections $\Delta n$, the long-wavelength modes with $\tau / \hbar \ll n_{0}$ obey linear, translationally invariant equations of motion and therefore behave independently. Let us analyse the motion of a single mode with frequency $\tau$ and amplitude $\chi_{\tau}$ :

$$
X(E)=\operatorname{Re} \chi_{\tau} \exp (\mathrm{i} E \tau / \hbar) .
$$

First, the viscous restoring force on the mode will be calculated. This force $F$ is defined by

$$
\left\langle\delta \chi_{\tau}\right\rangle=-\frac{F_{\tau}}{\nu} \delta \Gamma
$$

where $\Gamma$ is the fictitious time variable, and $\nu$ the viscosity. Then the diffusion constant $D_{\tau}$ of the mode will be calculated; this is defined by

$$
\left\langle\left|\delta \chi_{\tau}\right|^{2}\right\rangle=D_{\tau} \delta \Gamma
$$

where $\delta \chi_{\tau}$ is the drift in $\chi_{\tau}$ in a short time $\delta \Gamma$.

The force on a single particle is (from (2.12))

$$
F_{n}=F\left(E_{n}\right)=\kappa \nu \sum_{m \neq n} \frac{1}{E_{n}-E_{m}}=\kappa \nu \int_{-\infty}^{\infty} \mathrm{d} E \frac{n(E)}{E_{n}-E}
$$

so that, using (A2.2)

$$
\nu\langle\delta \chi(E)\rangle=F(E) \delta \Gamma=-\kappa \nu n_{0} \delta \Gamma \int_{-\infty}^{\infty} \mathrm{d} E^{\prime} \frac{1}{E-E^{\prime}} \frac{\mathrm{d} X\left(E^{\prime}\right)}{\mathrm{d} E^{\prime}} .
$$

Now substitute (A2.4) into (A2.8)

$$
\begin{aligned}
\operatorname{Re}\left(\delta \chi_{\tau}\right\rangle \exp (\mathrm{i} E \tau / \hbar) & =\operatorname{Re}\left(-\frac{\mathrm{i} n_{0} \tau \chi_{\tau}}{\hbar}\right) \int_{-\infty}^{\infty} \mathrm{d} E^{\prime} \frac{1}{E-E^{\prime}} \exp \left(\mathrm{i} E^{\prime} \tau / \hbar\right) \delta \Gamma \\
& =\operatorname{Re}\left(\frac{\mathrm{i} \kappa n_{0} \tau}{\hbar} \chi_{\tau} \delta \Gamma \exp (\mathrm{i} E \tau / \hbar)\right) \int_{-\infty}^{\infty} \frac{\mathrm{d} u}{u} \mathrm{e}^{\mathrm{i} u} \\
& =\operatorname{Re}\left(-\frac{\kappa n_{0} \tau}{\hbar} \exp (\mathrm{i} \varepsilon \tau / \hbar) \chi_{\tau} \delta \Gamma\right) \int_{-\infty}^{\infty} \frac{\sin u}{u} \mathrm{~d} u
\end{aligned}
$$

so that

$$
\left\langle\delta \chi_{\tau}\right\rangle=\frac{F_{\tau}}{\nu} \delta \Gamma=-\frac{\pi \kappa n_{0} \tau}{\hbar} \chi_{\tau} \delta \Gamma
$$

Note that this restoring force is proportional to the amplitude $\chi_{\tau}$ of the mode.

Now consider the diffusive behaviour of the mode. The diffusive displacement $\delta X\left(E_{n}\right)$ of the $n$th energy level in time $\delta \Gamma$ is equal to the matix element $A_{n n}$. The diffusive motion is therefore described by the sum $f(E)$ introduced in $\S 3$

$$
\begin{aligned}
f(E) & =\sum_{n} A_{n n} \delta_{\varepsilon}\left(E-E_{n}\right) \\
& =\sum_{n} \delta X\left(E_{n}\right) \delta_{\varepsilon}\left(E-E_{n}\right) .
\end{aligned}
$$


The sum rule (3.20) shows that $\delta X(E)$ is the sum of a discrete set of modes corresponding to the periodic orbits

$$
\delta X(E)=\sum_{j} \delta \chi_{j} \exp \left(\mathrm{i} E \tau_{j} / \hbar\right)
$$

where $\delta \chi_{j}$ is the amplitude of the diffusive motion of frequency $\tau_{j}$. Modes with frequencies which do not correspond to periodic orbits are not driven by the Brownian motion, and (A2.10) shows that their amplitude decays to zero. Comparing (A2.11) and (A.2.12) with (3.20) we have, taking the limit $\varepsilon \rightarrow 0$

$$
\begin{aligned}
f(E) & =\sum_{j} \delta \chi_{j} \sum_{n} \exp \left(\mathrm{i} E_{n} \tau_{j} / \hbar\right) \delta_{0}\left(E-E_{n}\right) \\
& =n_{0} \sum_{j} \delta \chi_{j} \exp \left(\mathrm{i} E \tau_{j} / \hbar\right) \\
& =\sum_{j} \alpha_{j} \bar{A}_{j} \exp \left(\mathrm{i} \sigma_{i}^{0} / \hbar\right) \exp \left(\mathrm{i} E \tau_{j} / \hbar\right)
\end{aligned}
$$

where $\sigma_{j}=\sigma_{j}^{0}+E \tau_{j}$, so that

$$
\delta \chi_{j}=\frac{\alpha_{j} \bar{A}_{j}}{n_{0}} \exp \left(\mathrm{i} \sigma_{j}^{0} / \hbar\right) .
$$

Using this result and equations (3.9) (3.28) and (2.5), we find

$$
\begin{aligned}
\left\langle|\delta \chi|^{2}\right\rangle & =\alpha_{j}^{2}\left\langle\bar{A}_{j}^{2}\right\rangle / n_{0}^{2} \\
& =2 \pi \hbar \alpha_{j}^{2} \sigma^{2} / n_{0} \tau_{j} \\
& =\frac{2 \pi \hbar \kappa}{n_{0}} \frac{\alpha_{j}^{2}}{\tau_{j}} \delta \Gamma
\end{aligned}
$$

so that the diffusion constant for the mode, $D_{j}$, is given by

$$
\left\langle\left|\delta \chi_{j}\right|^{2}\right\rangle=D_{j} \delta \Gamma=\frac{2 \pi \hbar \kappa}{n_{0}} \frac{\alpha_{j}^{2}}{\tau_{j}} \delta \Gamma .
$$

These results can also be expressed in terms of the amplitudes $\alpha_{j}$ of the periodic density fluctuations, instead of those of the displacement modes. Using (A2.2), the relationship between these coordinates is

$$
\alpha_{j}=\left(\mathrm{i} n_{0} \tau_{j} / \hbar\right) \chi_{j}
$$

In terms of the $\alpha_{j}$ coordinates, the relaxation and diffusion equations (A2.10) and (A2.16) become

$$
\left\langle\delta \alpha_{j}\right\rangle=-\left(\pi \kappa n_{0} \tau_{j} / \hbar\right) \alpha_{j} \delta \Gamma
$$

and

$$
\left\langle\delta \alpha_{j}^{2}\right\rangle=\left(2 \pi \kappa n_{0} / \hbar\right) \alpha_{j}^{2} \tau_{j} \delta \Gamma
$$




\section{References}

Balian R and Bloch C 1972 Ann. Phys., NY $6976-160$ 1974 Ann Phys., NY 85 514-45

Berry M V 1983 Chaotic Behaviour of Deterministic Systems, Les Houches Lectures vol 36, ed G Iooss, R H G Helleman and R Stora (Amsterdam: North-Holland) pp 171-271 1985 Proc R. Soc. A 400 229-51

Berry M V and Tabor M 1976 Proc. R. Soc. A 349 101-23

- 1977a Proc. R. Soc. A 356 375-94

- 1977b J. Phys. A: Math. Gen. $10371-9$

Berry M V and Wilkinson M 1984 Proc. R. Soc. A 392 15-43

Bohigas O, Giannoni M J and Schmidt C 1984 Phys. Rev. Lett. 52 1-4

Dyson F J 1962a J. Math. Phys. 3 140-56

1962b J. Math. Phys. 3 1191-8

Dyson F J and Mehta M L 1963 J. Math. Phys. 4 701-12

Gutzwiller M C 1967 J. Math. Phys. 8 1979-2000

1969 J. Math. Phys. 10 1004-20

1970 J. Math. Phys. 11 1791-806

1971 J. Math. Phys. 12 343-58

- 1980 Phys. Rev. Lett. 45 150-3

Groenewold H J 1946 Physica 12 405-60

Hannay J H and Berry M V 1980 Physica 1D 267-90

Hannay J H and Ozorio de Almeida A M 1984 J. Phys. A: Math. Gen. 17 3429-40

Hietarinta J 1984 J. Math. Phys. 25 1833-40

Moyal J E 1949 Proc. Camb. Phil. Soc. Math. Phys. Sci. 45 99-124

Pechukas P 1983 Phys. Rev. Lett. 51 943-6

Porter C E 1965 Statistical Theories of Spectra: Fluctuations (New York: Academic)

Richens P J and Berry M V 1981 Physica 2D 495-512

Weyl H 1927 Z. Phys. 46 1-46

Wilkinson M 1986 Physica 21D 341-54

— 1987a J. Phys. A: Math. Gen. $20635-45$

1987b J. Phys. A: Math. Gen. 20 2415-23 